\title{
Contexto sociocultural y alimentario de mujeres con embarazos de riesgo
}

\author{
The socio-cultural and eating aspects \\ of women experiencing risk pregnancies
}

Reyna Sámano Sámano ${ }^{1}$

Estela Godinez Martínez ${ }^{1}$

Irma Romero Pérez ${ }^{1}$

Georgina Sánchez Miranda ${ }^{1}$

José Manuel Espíndola Polis ${ }^{1}$

Mayra Lilia Chávez Courtois ${ }^{1}$

\footnotetext{
${ }^{1}$ Instituto Nacional de Perinatología Isidro Espinosa de los Reyes. Montes Urales 800/Torre de Investigación $2^{\circ}$ Piso, Col. Lomas Virreyes. 11.000 México DF. Dra. Mayra Lilia Chávez Courtois courml@yahoo.com.mx
}

\begin{abstract}
To promote a healthy diet, the dietary guidance should consider socio-cultural factors as they influence both the food standards and preferences of each population. The aim of this study was to describe the socio-cultural contexts of pregnancy and feeding in the domestic arena, using $a$ qualitative approach. In-depth interviews were conducted using the data saturation technique with six women experiencing high-risk pregnancy. The interviews were transcribed and analyzed in accordance with the Bardin method and the IT Atlas program. The following aspects were identified: uncertainty due to unplanned pregnancy and limited partner support; a few dietary changes to improve health; perception of bad and good feeding habits, although it did not comply with the characteristics of a good diet; omission of fruit and vegetable group from dinner. The people in charge of preparing and serving food were not always the pregnant woman; food products perceived as being harmful were consumed and beneficial products were rarely consumed. This highlighted the need to train professionals to consider the socio-cultural context when providing dietary guidance.
\end{abstract}

Key words High-risk pregnancy, Feeding, Sociocultural context
Resumen Para favorecer una alimentación correcta la orientación alimentaria debe considerar factores socioculturales que condicionen patrones $y$ preferencias del consumo de alimentos propios de cada población. El objetivo de esta investigación fue describir el contexto sociocultural del embarazo y su alimentación en el ámbito doméstico, mediante un enfoque cualitativo. Se realizaron entrevistas en profundidad a 6 mujeres con embarazo de alto riesgo bajo la técnica de saturación de la información. Las entrevistas se transcribieron y analizaron de acuerdo a Bardín con ayuda del programa Atlas TI. Se identificó: incertidumbre por el embarazo no planeado y poco apoyo de la pareja; ciertos cambios en la alimentación para mejorar la salud; percepción de una mala $y$ buena alimentación, aunque ésta no cumplía con las características de una dieta correcta; omisión de la cena y del grupo de verduras y frutas. Las encargadas de preparar y servir los alimentos no siempre fueron las mujeres embarazadas; los alimentos percibidos peligrosos fueron consumidos y los benéficos presentaron consumo escaso. Resaltó la necesidad de capacitar a los profesionales de la salud para considerar al contexto sociocultural en la orientación alimentaria que proporcionen.

Palabras clave Embarazo de alto riesgo, Alimentación, Contexto sociocultural 


\section{Introducción}

A lo largo de la historia mundial, la manifestación y por ende la comprensión del embarazo ha estado inserta en el contexto sociocultural que conlleva creencias y costumbres propias; por ejemplo en algunas sociedades occidentales si una mujer en matrimonio o juntada no se embaraza -ya sea por decisión propia o problemas biofisiológicos de la pareja- puede dar la impresión de no cumplir con sus responsabilidades matrimoniales culturalmente dadas, por lo cual en ocasiones tiende a ser objeto de críticas y/o lástimas $^{1}$; de ahí la relevancia de comprender que el embarazo por ser una exigencia sociocultural juega un papel importante en la actualidad. Otro elemento en el proceso del embarazo es lo relacionado con la alimentación que practica la gestante. Se sabe que el tipo y calidad de alimentos también responden a situaciones del contexto inmediato: socioculturales, económicas, psicológicas, biológicas ${ }^{2}$; así durante el embarazo se ha acostumbrado que ni por temor, pena o vergüenza las mujeres embarazadas omitan sus antojos de comida, golosinas entre otras, debido a que al no cumplir con esa necesidad se cree que pondrían en riesgo la salud materna-fetal ya que es un requerimiento del niño a través de la madre ${ }^{3}$.

El control de la alimentación durante el embarazo y otras etapas de la vida está regida por percepciones negativas o positivas de los alimentos a través de la asignación de atribuciones asignadas a los alimentos como permitidos y prohibidos $^{4,5}$, teniendo repercusiones sobre su estado nutricio y calidad de vida, como ejemplo se documentó que cerca de la mitad de una muestra de 300 dominicanas refirió tener algún prejuicio contra alimentos como la fruta o los huevos durante sus embarazos ${ }^{5}$. Por tal, no siempre se logran los ideales de una dieta correcta, desde el punto de vista de especialistas de la nutrición ${ }^{6}$. Así, se ha encontrado que mujeres gestantes obesas y no obesas no consumen los tres grupos de alimentos recomendados ${ }^{7}$; debido a que las preferencias y aversiones determinan prácticas de adquisición, preparación y consumo de alimentos ${ }^{8}$.

En México las embarazadas en se han considerado vulnerables por su mala alimentación y por no programar sus embarazos ${ }^{9}$, iniciándolos con reservas inadecuadas de nutrimentos como hierro y calcio ${ }^{10}$ pudiendo desarrollar problemas de nutrición maternos y fetales en mayor frecuencia y severidad. De este modo, es necesario el apoyo de instituciones sociales que faciliten la reducción de la anemia nutricional y la promo- ción de conductas que no afecten su alimentación con relación a la dieta familiar ${ }^{11}$, respetando sus creencias y significados a los alimentos para lograr un mayor apego a una alimentación correcta que les permita llevar un embarazo saludable y así mejorar su pronóstico; además se podría reducir el gasto familiar y gubernamental destinado al control de afecciones como la diabetes e hipertensión y sus complicaciones durante la gestación y parto ${ }^{12}$.

En el ámbito doméstico dentro del proceso de la alimentación, se ha documentado que las mujeres independientemente de su estado reproductivo son las encargadas desde la compra hasta lavado de utensilios ${ }^{13}$; ahora pensemos en una mujer embarazada que también tiene participación en el trabajo remunerado ${ }^{14}$, con esto se observa una carga de trabajo adicional importante; exponiéndolas a un aumento de desgaste físico y emocional por la responsabilidad de aportar ingreso al gasto familiar, adicional al trabajo doméstico y la gestación, por lo que cualquier actividad física las obliga a adoptar posturas incómodas de riesgo ${ }^{15}$, implicando un mayor gasto de energía ${ }^{16}$.

Las mujeres con embarazos de alto riesgo a pesar de ser vulnerables han sido poco estudiadas. Muchas de ellas mueren por no tener información, ni un entorno social que apoye una alimentación sin riesgos a la salud. En ese sentido, es necesario considerar su cultura alimentaria, en especial la familiar, pues ésta incide en sus hábitos alimentarios. Así, es preciso conocer cuáles son los alimentos que las gestantes prefieren consumir en sus tiempos de comida y con ello identificar los efectos maternos adversos que directamente repercuten en sus hijos como el retardo en el crecimiento y bajo peso al nacer, con un crecimiento postnatal inferior al que su potencial genético lo permite, en contraste, el exceso de peso puede provocar diabetes ó hipertensión maternas y en los hijos macrosomía y/o prematuros ${ }^{17}$. Por lo anterior, el objetivo de esta investigación fue describir parte del contexto sociocultural del embarazo y su alimentación en el ámbito doméstico, mediante un enfoque cualitativo.

\section{Material e métodos}

La presente investigación cualitativa forma parte del proyecto intitulado Estudio piloto sobre factores socioculturales del cuidado prenatal asociados a la mortalidad materna; aceptado por los Comités de Ética e Investigación del Instituto Nacional 
de Perinatología INPer. Desarrollado de 2010 a 2011. La investigación cualitativa permitió identificar experiencias particulares relativas al embarazo y alimentación, comprendiendo en la medida de lo posible su realidad social ${ }^{17}$.

La investigación se realizó con usuarias del INPer, el cual es un Instituto de Salud que se caracteriza por atender afecciones en la salud reproductiva sobre todo de las mujeres, siendo una de ellos, los embarazos de alto riesgo, infertilidad, entre otros. Se contó con la información de 6 mujeres con el criterio básico de inclusión: embarazo de riesgo ${ }^{18}$. Fueron gestantes que por diversas circunstancias no lograban embarazarse (dos de ellas), tenían pérdidas gestacionales recurrentes (tres de ellas) o bien que padecían de alguna enfermedad previa al embarazo actual. Por los propios criterios de selección, se cubrió un perfil semejante entre las participantes con lo que el nivel de saturación de elementos básicos de la información ${ }^{19}$ se logró con seis participantes según los meta-temas de interés: en este caso la relación del contexto sociocultural y la alimentación, ver Tabla 1. El manejo de la información fue anónimo, confidencial y se utilizaron seudónimos. Todas las participantes firmaron carta de consentimiento informado en compañía de dos testigos, además, en el caso de las menores de edad, se solicitó la autorización y firma de sus padres o tutores. Todo el grupo de trabajo de esta investigación se estandarizó para la obtención de datos sobre el contexto de la gestación y alimentación durante el embarazo.

Se contó con la entrevista en profundidad bajo la técnica de saturación de la información de elementos básicos de la información, en la que se decide concluir con la invitación de participantes cuando el contenido de las narrativas se comienza a repetir, y del cual ya no se obtiene información relevante según el tema de interés de análisis. Las entrevistas se hicieron con una duración aproximada de dos horas y media, en dos a tres sesiones por informante; se realizaron en un espacio privado y se audiograbaron. El espacio y lugar lo decidió la participante para mayor comodidad de ella, de este modo, todas las participantes optaron por ser entrevistadas en su domicilio sin la presencia de familiares o amigos y en las fechas establecidas por ellas. Las entrevistas se transcribieron y fueron analizadas de acuerdo con Bardín ${ }^{20}$ en tres fases: la de preanálisis, la de exploración y la de interpretación de los resultados con ayuda del programa de análisis Atlas Ti. Las entrevistas fueron organizadas con las siguientes líneas: 1) Situación en la que se presentó la gestación; 2) Contexto alimentario durante la gestación: cambios en la alimentación, consumo de alimentos y alimentos benéficos y peligrosos para la gestación.

\section{Resultados}

\section{Incertidumbre del nuevo embarazo: eventos adversos previos y actuales}

Los resultados de acuerdo con las categorías de análisis establecidas encontradas en las entrevistas permitieron analizar que el contexto en que se presentó el embarazo fue de riesgos físicos

Tabla 1. Datos generales de las participantes.

\begin{tabular}{lllllll}
\hline & \multicolumn{1}{c}{ Sandra } & \multicolumn{1}{c}{ Alicia } & Eréndira & Nayeli & Edith & Luisa \\
\hline Edad (años) & 33 & 35 & 17 & 19 & 34 & 43 \\
EG & 34 & 30 & 32 & 29 & 29 & 30 \\
Escolaridad & Secundaria & Técnica & Secundaria & Secundaria & Técnica & Técnica \\
Edo. civil & Casada & Casada & Soltera & Casada & Soltera & Soltera \\
Religión & Mormona & Católica & Cristiana & Católica & Cristiana & Católica \\
Ocupación & Hogar & Hogar & Estudiante & Hogar & Comerciante & Empleada \\
Residencia & Edo. México & Edo. México & Cd. México & Edo. México & Cd. México & Cd. México \\
Gestación & G5 & G2 & G1 & G2 & G1 & G4 \\
Diagnóstico (s) & EMA,RH, & EMA, MU, & Adolescente. & Huevo muerto, & MU, Microadenoma EMA, 2 \\
& Antecedentes & Aborto, & & Pareja con & hipofisiario, & abortos, \\
& de DM. & Banda & & toxicomanía. & Ausencia riñón & Incompetencia \\
& & amniótica. & & & derecho. & itsmocervical \\
& & & & & con cerclaje. \\
\hline
\end{tabular}

EG: Edad gestacional en semanas; G: número de gestación; EMA: edad materna avanzada; RH: Factor sanguíneo negativo; MU: miomatosis uterina. 
importantes como enfermedades previas y presentes, pérdidas intercurrentes gestacionales, edad materna avanzada o menores de edad ... del otro bebé cuando me vine para'ca el doctor me mandó hacer un ultrasonido vaginal $y$... encontraron al niño ya muerto, fuera de la matriz. ..., por eso, con este nuevo embarazo a pesar de que bajé 6 kilos los primeros 4 meses...está el sentimiento de que vaya a pasar algo (Nayeli, 19 años). Desde el punto de vista social se observó el abandono de la pareja y negación al embarazo. No obstante, resaltó que la madre, hermanas, abuela o demás familia fueron quienes las apoyaron en ausencia de la pareja.

La incertidumbre del nuevo embarazo al menos para este grupo de mujeres se dio en tres sentidos, por un lado lo relacionado con la pareja, en el cual se evidenció un proceso de negación de la presencia del embarazo posterior al enojo con la pareja; además de incertidumbre de cómo informar a la familia del evento independientemente de la edad de la participante. Y por último sin ser el menos importante, la inseguridad al logro del hijo vivo... luego, luego me salió positivo, 'aauch!, bueno, pues ya ni modo', entonces si me dio un poquito de miedo; porque pues ya en el 2000 yo perdi a un bebé y en el 2003 fue el legrado, lo anhelaba... porque yo soy $\mathrm{RH}$ negativo y con dos hijos vivos. (Sandra, 33 años).

La inseguridad del logro del hijo vivo se relacionó con la condición de salud y eventos reproductivos en la mujer. Se observaron condiciones concernidas con el sentimiento de culpa por la presencia de abortos voluntarios o involuntarios, que las llevaron a la resignación de la continuación del nuevo embarazo. Por ejemplo en el caso de Nayeli, la pérdida de un hijo y su condición de salud - pérdida de $6 \mathrm{~kg}$ en los primeros cuatro meses del actual embarazo - provocó en ella miedo en su proceso reproductivo.

El caso de Sandra de 34 años de edad, es una muestra clara de la resignación y la presencia de miedo, pues ante la noticia se sorprende y preocupa al mismo tiempo porque ha tenido pérdidas gestacionales, tiene limitaciones económicas, ya tiene dos hijos vivos; que si bien cuenta con el apoyo de su pareja, la salud de ésta no es la óptima y está en riesgo alto.

Es relevante mencionar que el embarazo fue visualizado como una obligación de la mujer ante la sociedad, aún en contra de la integridad de la propia salud y del abandono de la pareja como lo refirió Sandra. Finalmente todas identificaron el embarazo como inesperado, no planeado, pero al final aceptado. me siento realizada como mujer, me siento completa también como mujer. Aunque sea sin pareja (Luisa, 43 años)

Podemos ver que para este grupo la incertidumbre de la presencia del nuevo embarazo es un conjunto de situaciones conjugadas unas con otras, resumiéndolo en la triada: inestabilidad de la pareja-con condición de salud desfavorableeventos reproductivos adversos.

\section{La alimentación durante el embarazo cambia siempre y cuando haya elementos que la fundamenten}

En la alimentación se observaron dos tendencias durante el embarazo: 1) Cambios, como lo refirió una de las participantes ...tener que comer menos, quité grasas, quité dulces, quité irritantes... porque estaba subiendo mucho de peso, ya como menos, si antes me comía 4 ó 5 tortillas ahora ya nada más me como una... jsi le he bajado!, si me agarraba a comer frutas de una manzana dos manzanas nada más me como una pero me da un montón de hambre (Sandra, 33 años). Ella adoptó estrategias para mejorar el pronóstico de su gestación, que implicaron la eliminación de azúcares simples y grasa, o la disminución de la cantidad de alimentos como la tortilla y el pan e incorporación de fruta, con el argumento de que estaba aumentando peso de más y ponía en riesgo la integridad de su salud. Si bien, sólo una de ellas se sometió a un plan de alimentación sugerida por los médicos, se pudo documentar que con éste ella se quedaba con hambre, lo que le implicó no poder seguirla por mucho tiempo, es decir el fracaso en el plan de alimentación. 2) En contraste, se observó que no siempre cambia significativamente la dieta, ... pero la alimentación... pues si prácticamente sigue siendo... la misma que hemos comido durante toda nuestra vida, sopa, arroz, el guisado, agua, refresco trato, ;trato!, de evitarlo, porque está uno tan acostumbrado que nunca, o sea, siempre el refresco aquí en tu mesa si no uno no está a gusto ¿no?, pero, pero si este, yo siento que mi alimentación es bueno, yo la veo bien ¿no? (Edith, 34 años). En esta informante se reconoce que su dieta sigue siendo la misma incluso considera la necesidad de abandonar el refresco, pero no lo logró, porque los demás integrantes de su familia lo acostumbran y siempre está disponible, en otras palabras, la familia permea en gran medida el consumo o no del producto. Con todo y eso se presentó el fenómeno de una autopercepción de practicar una buena alimentación entre las que no cambiaron. 
Cabe señalar que el período del embarazo para algunas de ellas fue una oportunidad para modificar la alimentación ya sea para mejorarla o para quedarse con hábitos alimentarios inadecuados; repercutiendo directamente en la salud de la madre y el bebé. De esta forma se observó que los azúcares se consumieron de más, sobre todo cuando tenían hambre.

...y este yo he comido más,... como más de la cuenta, yo creo que tengo como unos 13 kilos arriba ya de mi peso,... hay días que sí como y como y como y quiero estar comiendo tanta comida como chatarra ¿no?, hay veces que se me antoja una galletita, que ... un helado, que una paleta, que esto o lo otro... iponme un kilo de tortillas y un kilo me aviento y en las noches ponme dos tres piezas de pan, un paquete de galletas me lo llego a comer yo sola y un refresco... eso si hay veces que igual se me antoja mucho una taza de café, y si se me antoja ¡va pa' dentro! ¿no? (Edith, 34 años)

Contrario a lo anterior, durante el proceso de la gestación el consumo de verduras y frutas aumentó en mayor frecuencia y se dejó ver en algunos casos el apoyo de la familia para lograrlo.

Las verduras, ahorita me está haciendo sopas ya sea de lentejas o sopa de pasta con espinacas, con chayotes, con papas, con zanahorias esas son las verduras que he consumido más a raíz del embarazo en realidad. De las frutas, realmente yo no soy muy afecta a las frutas casi no, sí me gustan pero casi no las consumo pero ahorita consumo papaya, manzana, manzana y pues la fruta de temporada generalmente. (Luisa, 43 años)

\section{Percepción de la alimentación y consumo de alimentos}

Relacionado con lo anterior, en la autopercepción de la alimentación se identificaron dos categorías: 1) como bien, 2) no como bien. En esta última incluso se reconoce por qué no come bien; en la primera, a pesar de que consideran comer bien no necesariamente cumplen con las características de una dieta correcta ${ }^{6}$.

Autopercepción de comer bien

...yo la veo bien (alimentación), este y esa es la forma en la que te digo a veces como mucho, a veces...dejo de comer así este, como bien, como bien poquito pero eso es así, esporádico, porque la mayoría de las veces si me paso, y cuando me de hambre trato de comer este, nooo tanta chatarra no pero si o sea ¿no?, yo siento que mi alimentación es buena, yo la veo bien ¿no?(Edith, 34 años)

Autopercepción de comer no bien
No, porque comer bien sería comer más ensaladas, más sopas, menos coca, menos chile pero no, no me alimento bien." (Nayeli, 19 años)

La percepción de las mujeres respecto al hecho de comer sanamente o no, está relacionado con los tipos de alimentos que consumen y no tanto por la cantidad de los mismos; por ejemplo en ambos casos hacen referencia del producto chatarra, que es considerado un alimento no favorable durante el embarazo. Pero cuando analizamos desde el enfoque el concepto de consumo de alimentos, vemos que sí hacen referencia a la cantidad de éstos y que este último también define el tipo de alimento. Por ejemplo en el caso de Sandra, quien se sometió a un plan de alimentación expedido por profesionales de la salud, sin embargo, ella mencionó que no lo siguió al pie de la letra porque la cantidad de alimentos que le recomendaron era muy poca, lo que le provocaba quedarse con hambre y comer cosas que no debía. Además, se observó que ninguno de los casos se acostumbraba el consumo de pescado.

Con lo anterior podemos decir que la percepción de un buen comer está dado por el tipo de alimentos, aunque el consumo de ellos (tipo y cantidad) es determinante en la práctica para un comer bien o no según la NOM-043-SSA2-2005 de México ${ }^{6}$.

Uno de los hallazgos interesantes fue que dos casos omitían la cena y que de los grupos de alimentos incluidos en todos los tiempos de comida los más constantes fueron los cereales y alimentos de origen animal, con ausencia invariable de las verduras y frutas. Una de las razones por las que se excluía la cena, fue por la sensación de sentirse muy llenas y para evitar algún malestar nocturno, pero además se dejó ver que no se acostumbraba ese tiempo de comida desde antes del embarazo. Es decir, el embarazo no modificó la costumbre en los tiempos de comida.

...procuro no cenar porque... Trato de tomar un plátano, leche con corn flakes si siento hambre en la noche pero no, no ceno. De por sí, no cenaba antes. (Luisa, 43 años)

Para conocer las razones por las que algunas participantes excluyen el consumo de verduras como posible respuesta a dicha conducta, ${ }^{21}$ se indagó en la creencia respecto a verduras peligrosas en el embarazo, y se encontró que para este grupo todas las verduras son buenas y que no hay razón para no consumirlas, pero a pesar de lo manifestado su consumo es escaso y cuando las comen lo hacen preparadas con grasa.

...ninguna verdura se debe evitar...Al vapor con mantequilla... Las calabazas con crema, los 
bisteces en chile habanero, son lo que más se come. (Nayeli, 19 años)

\section{No siempre son las mismas las que cocinan $\mathrm{y}$ sirven de comer}

Uno de los eslabones del proceso de la alimentación es la preparación y distribución de alimentos o comida, en este tópico se observaron varias tendencias al preparar la comida o el orden en que se sirven las cantidades, o la distribución de los lugares a la hora de sentarse a consumir los alimentos, así se identificaron 3 constantes en la preparación.

a) Las que siempre cocinan

... yo soy la que prepara, yo soy la que hace la comida... Yo cocino (Edith, 34 años)

b) Las que ocasionalmente preparan y sirven los alimentos

Yo, o a veces cuando si me siento mal pues la que sirve es mi hija es la que da de comer, cuando yo no me siento así con ánimos o que me siento cansada así, pues ella es quien nos da de comer, o sea, yo hago la comida pero ya cuando es la hora de comer, este o igual yo sirvo y ella calienta las tortillas (Sandra, 33 años)

c) Las que no cocinan

Ella [su madre] los compra [alimentos] y también los prepara o mi esposo, también los compray los prepara. (Nayeli, 19 años)

La distribución de alimentos presentó dos tendencias

1) Las que se encargan de servir y no hay un orden al momento de sentarse.

La comida y la cena yo, el desayuno mi mamá...porque se va mi hermano a la universidad, mi papá al trabajo entonces se van o se sirven ellos o se levanta mi mamá, todos parejos...se sientan en donde quieran y se les sirve a todos por igual. (Nayeli, 19 años)

2) En la que cada uno de los integrantes de la familia se sirve sus alimentos sin un orden al momento de sentarse.

... a todos iguales, cada plato se pone en su lugar y ya cada quien se sienta y a comer. (Sandra, 33 años).

Los alimentos peligrosos y benéficos para el embarazo, ¿se consideran en la práctica?

\section{Peligrosos}

Un aspecto notable fue identificar los alimentos que las participantes refieren como peligro- sos para el embarazo, los más constantes fueron las bebidas de dieta, sal, picante, pan, tortilla, refresco y café; sin embargo, aún teniendo el conocimiento se dejó ver que algunas participantes confirmaron su consumo a pesar de saber que ello puede afectar de forma negativa su embarazo. Dicha referencia de los alimentos tuvo como preámbulo tanto las indicaciones dadas del personal de salud como el contenido empírico, es decir, lo que les dicen y aprenden de sus familiares o personas cercanas con relación a ese tipo de sustancias o alimentos, como se puede observar en el Cuadro 1.

No obstante, se siguen consumiendo algunos alimentos que refirieron como peligrosos, por ejemplo el café y el picante.

\section{Benéficos}

Contrario a lo anterior, se desarrolló el tópico de alimentos que las informantes refieren pueden beneficiar el embarazo, los más mencionados fueron, el pescado y las verduras, sin embargo, su consumo fue nulo y escaso respectivamente, aún teniendo presente los beneficios que les traería su consumo, como se puede ver en el Cuadro 1.

Por otro lado, el término frío caliente no tuvo un significado definido, incluso hubo quien mencionó que sólo es un mito. Las demás refirieron no saber nada al respecto.

Los alimentos calientes te provocan más... mareo... ganas de volver el estómago... en los tres primeros meses, me daba mucho asco lo caliente me tranquilizaba con agua fría, frutas, algo frío porque lo caliente me ino!, era un marearse, a lo mejor tiene algo que ver las hormonas y ya la gente lo hizo mito. (Alicia, 35 años)

\section{Para una buena producción de leche, ¿qué se sabe?}

En otro tema, se exploraron las prácticas que realizaban para una producción adecuada de leche y se tuvo el aporte de que tomando mucha agua y comiendo bien se podría producir suficiente leche.

No, nada más que mucha agua, que tome muchos líquidos (Nayeli, 19 años)

Bueno pues yo sé que uno debe tener buena alimentación más que nada por la leche, para no subir de peso y todo eso... Pues yo me acuerdo... puro pollo, no irritantes,... un atole de masa sin colar según para la leche (Sandra,33 años) 
Cuadro 1. Alimentos peligrosos y benéficos durante el embarazo según las participantes.

\begin{tabular}{|c|c|c|}
\hline Peligrosos & Motivos & ¿Los consume? \\
\hline Café & Inhibe la absorción de no sé qué (Luisa, 43 años) & Si \\
\hline Sal & Me voy a hinchar (Eréndira, 17 años) & Como menos \\
\hline Picante & $\begin{array}{l}\text { No me hace bien... les nacen puntitos rojos a } \\
\text { los niños, luego se les quita (Nayeli, } 19 \text { años) }\end{array}$ & No debería \\
\hline Gaseosa & Por mis riñones... me hace mal (Nayeli, 19 años) & No la puedo dejar \\
\hline $\begin{array}{l}\text { Galletas, dulces, } \\
\text { refrescos }\end{array}$ & $\begin{array}{l}\text { Tengo la azúcar alta, puedo arriesgar que mi hijo } \\
\text { pueda llegar a desarrollar la diabetes (Sandra, } 33 \\
\text { años) }\end{array}$ & Sigo comiendo lo que no debo \\
\hline Carnes & $\begin{array}{l}\text { Traen toxinas y un montón de hormonas hace } \\
\text { daño (Edith, } 34 \text { años) }\end{array}$ & Procuro no comerla \\
\hline \multicolumn{3}{|l|}{ Benéficos } \\
\hline Pescado & $\begin{array}{l}\text { Pues yo digo que el número uno es el pescado, el } \\
\text { número } 2 \text { el pollo, y es el que más comi (Alicia, } 35 \\
\text { años) }\end{array}$ & No \\
\hline Todas las verduras & todas, todas te ayudan (Nayeli, 19 años) & No siempre \\
\hline Huevo & $\begin{array}{l}\text { ayuda mucho con el ácido fólico, es lo que he } \\
\text { escuchado (Luisa, } 43 \text { años) }\end{array}$ & Si \\
\hline
\end{tabular}

\section{Discusión}

La presente investigación cualitativa proporciona información del contexto particular de un grupo de mujeres con embarazos considerados de alto riesgo $^{18}$ por las propias características físicas y sociales en las que se encontraban al momento de la entrevista. En primer lugar, se observó un contexto del embarazo adverso, en condiciones fisiológicas de alto riesgo con predominio embarazos no planeados, lo que se ha reportado en otras investigaciones como una situación frecuente ${ }^{9}$, con repercusiones fisiológicas y psicológicas que las pueden conducir a un estado de depresión con serias consecuencias ${ }^{21}$ desde discrepancias hasta separación de la pareja ${ }^{22}$ con lo que el apoyo de ésta se llega a perder. Si bien la participación de la pareja fue limitada en nuestra investigación, se debe señalar que esto no es nuevo, dado que en eventos de este tipo se involucran situaciones culturales y sociales, asignando la maternidad como un evento propio de la mujer ${ }^{23}$, consecuencia en- tre otras razones socioculturales por la falta de involucramiento del hombre.

En nuestra investigación se consideró que las mujeres siguen atribuyendo como un deber el tener un hijo, ya que con eso se cumplirá su anhelo de ser una mujer realizada coincidiendo con Foster $^{1}$ a expensas de su propia vida y exponiéndolas a preocupaciones, requiriendo así del apoyo y comprensión de los profesionales de la salud o de la propia familia para salir de su problemática y disminuir los riesgos propios de su diagnóstico ${ }^{24}$.

Se observó que 4 de 6 mujeres reportaron algún tipo de cambio en su alimentación, sobre todo disminuyendo azúcares y/ó lípidos, pero cabe señalar que algunas mencionaron que omitían la cena desde antes de que se embarazaran, situación que mantuvieron. Se les dificultó la eliminación por completo del refresco, pan o golosinas; con ello se reitera la necesidad de considerar que la alimentación es un proceso en el cual interfieren diversas dimensiones sociales y una de ellas es la cultura alimentaria que comparte toda fa- 
milia o grupo con el que se vive, en la que para que un plan de alimentación funcione es necesario considerar las dimensiones sociales de la alimentación, de otra forma serán poco exitosos los planes de alimentación, por ejemplo en la práctica diaria de nutricionistas-dietistas, los pacientes tienen que crear situaciones en las que se prepara comida "especial para el enfermo" "diferente para la familia" lo que provoca que el paciente se ubique en cierta forma fuera del entorno social y cultural, es decir genera una ruptura cultural2 ${ }^{25}$, teniendo como resultado el abandono de la dieta, en el caso de nuestras participantes refirieron que cuando se ponían a dieta - recomendada por los profesionales de la salud - se quedaban con hambre y eso ocasionaba que para satisfacerla comieran cualquier golosina y con ello se obtenía el riesgo de complicar su panorama desde el punto de vista fisiológico, alterando los parámetros establecidos como normales para esta población, sobre todo en ellas con embarazos de alto riesgo. Los cambios en la alimentación fueron dirigidos según las costumbres de cada seno familiar, sean adecuados o no de acuerdo con los lineamientos normativos de la nutrición ${ }^{6}$. Así es como se observó que si no se acostumbraba cenar desde antes del embarazo, esto mantenía durante la gestación, con los argumentos de presentar malestar nocturno. En este sentido, es necesario destacar que para este grupo el embarazo no fue un condicionante para los cambios alimenticios (incluyendo los tiempos) pero sí las costumbres alimentarias familiares, en ese sentido podemos decir que la salud se constituía cubriendo las exigencias sociales, superando al mínimo el grado de funcionamiento orgánico que le permita su desarrollo pleno ${ }^{26}$.

En esta investigación también se lograron identificar los alimentos peligrosos según las percepciones de las embarazadas, siendo los irritantes los más citados, coincidiendo con dos estu$\operatorname{dios}^{5,27}$, sin embargo; también se corroboró que a pesar de que contaban con la información relativa al peligro de los alimentos, no se dejaron de consumir del todo por ser parte de sus alimentos cotidianos, con ello se reitera que el entorno es determinante para lograr o no el cese en su consumo. Diferente a lo reportado ${ }^{5,26}$ en nuestra investigación el conocimiento de los alimentos considerados como peligrosos no determinaron su consumo.

Contrario a lo anterior, los alimentos citados como benéficos fueron las verduras, el pescado y las frutas en mayor frecuencia, no obstante, no fueron consumidos del todo por las participan- tes, por el hecho de que no formaban parte de su dieta habitual o por economía. Sin embargo, una participante refirió que todo es bueno durante el embarazo, siempre y cuando sea con moderación, de esta forma ella no se quedaría con las ganas de comerlo ni afectaría su salud. Con ello se reitera la necesidad de que los profesionales de la salud consideren la percepción y consumo que las mujeres tienen respecto a los alimentos benéficos o peligrosos ${ }^{27}$, sobre todo que ambos se derivan de los hábitos alimentarios y costumbres según el contexto propio cuando se implementen planes de alimentación, esto para obtener mejores resultados acorde con las metas establecidas. Con lo anterior se ve claramente la urgencia de estrategias multidisciplinarias que identifiquen - no evalúen - de manera objetiva la alimentación, que incluyan preceptos culturales, sociales, disponibilidad y accesibilidad en torno a los alimentos, por ejemplo se reconoció al pescado como benéfico, pero por economía se prefería otro alimento de origen animal como el pollo, en este sentido, se debe destacar que a largo plazo la economía familiar puede traer como consecuencia "la costumbre" de comer siempre pollo y casi nunca el pescado fresco a pesar de sus beneficios o riesgos ${ }^{28}$ a la salud en población gestante mexicana.

Se observó que si bien, la mayoría de las participantes refirió haber aumentado el consumo de frutas y verduras, en los recordatorios se reflejó otra cosa, debido a que incorporaron estos alimentos a su dieta familiar, pero de acuerdo con los lineamientos establecidos como idóneos no lograron cubrir lo recomendado para este tipo de población ${ }^{6}$, aún así, consideramos que este es un primer paso para lograrlo y que se requiere de más atención de parte de los profesionales de la salud considerando siempre la cosmovisión de las pacientes ${ }^{29}$. Por ello, la etapa del embarazo es un buen momento para establecer una mejor alimentación para la mujer, su hijo y toda la familia; dado que las participantes determinaron autopercepciones heterogéneas de su alimentación y con ello la pauta de mejorarla porque ya cuentan con un autodiagnóstico, es decir una puerta parcialmente abierta en la que si oportunamente se interviene desde el punto de vista alimentario, se podrían prevenir nacimientos prematuros, pérdidas gestacionales, el desarrollo de diabetes gestacional y la retención de peso posparto, entre otras alteraciones.

Aunque se ha referido que la falta de voluntad y de poder adquisitivo para la compra de alimentos son las limitantes para una alimentación correcta $^{7}$; la primera se puede deber a que 
no se cuenta con la información suficiente o bien su contexto sociocultural no permite generar la convicción del cambio en la alimentación, así las mujeres requieren mayor información sobre su alimentación ${ }^{4}$, sobre todo, considerando que están en un momento en el que desean preservar la vida; en el caso de nuestra población de estudio se tiene la oportunidad de orientarlas al asistir mensualmente a sus consultas prenatales y así se podría implementar de forma adecuada y completa la consulta nutricia, en la que se ha comprobado que disminuye el riesgo de complicaciones secundarias a la hipertensión, considerada como una de las causas de preeclamsia ${ }^{30,31}$.

En este trabajo se observó que no hubo una distribución de alimentos asignada por género; ya que algunas de nuestras participantes viven con sus padres o hermanas y la distribución y asignación de lugares en la mesa es por otras razones, tales como quien llegue primero o quien tenga más hambre, por ello se puede decir que cuestiones económicas más que de género son las determinantes en lo que se come ${ }^{32}$. En contraste, con la preparación de los alimentos la mujer sigue siendo la encargada de ello, a menos que se enferme, similar a lo documentado por Gracia-Arnaíz en $2009^{14}$, lo que la expone a mayor estrés cuando tiene que reposar por la pro- pia gestación de alto riesgo ${ }^{15}$, al tener que delegar esa responsabilidad ${ }^{24}$.

Por otro lado, en nuestro grupo de participantes la clasificación de alimentos fríos y calientes no es clara y ésta no interviene directamente en su consumo, ya que se especificó que la cantidad de comida es la que afecta la salud, más que los propios alimentos e incluso algunas lo reconocieron como mitos, contrario a lo hallado en población rural de México ${ }^{32}$ lo que implica la pérdida de ciertos saberes populares en torno a los alimentos en nuestro grupo de estudio.

Para concluir, podemos decir que se requiere capacitar a los profesionales de la salud para la implementar planes de alimentación, en los cuales se considere tanto el diagnóstico como el contexto sociocultural ${ }^{25,33}$ de la embarazada. Pues se ha documentado que proporcionar planes de alimentación y consejería personalizada resulta mejor opción para que las pacientes entiendan y se adhieran más a las recomendaciones de los profesionales de la salud ${ }^{34}$, sobre todo durante el embarazo; lo anterior con el fin de que en ellas se logren prácticas de alimentación ad hoc y disminuya en la medida de lo posible la confusión ${ }^{35}$ que se tiene de la alimentación y sus cuidados durante la gestación para prevenir riesgos a la salud de la díada madre-hijo.

\section{Colaboradores}

R Sámano, EG Martínez, IR Pérez, GS Miranda, JME Polis y MC Courtois participaron igualmente de todas las etapas de elaboración del artículo.

\section{Referencias}

1. Foster G. Folklore y costumbres del embarazo, nacimiento e infancia. In: Kenny M, Miguel JM, editores. La antropología médica en España. Barcelona: Anagrama; 1980. p. 250.

2. De Garine I. Antropología de la alimentación y pluridisciplinariedad. América Indígena 1988; XLVIII(3):635-650.

3. Rodríquez ME. Costumbres y tradiciones en torno al parto en el México Virreynal. Anuario de Estudios Americanos 2000; Tomo LVII(2):501-522.

4. Saidman S, Raele MG, Basile M, Barreto L, Mackinonn MJ, Poy MS, Terraza R, López LB. Knowledge, interests and beliefs on food and nutrition in pregnant women. DIAETA 2012; 30(139):18-27.

5. Sánchez-Brito L. Cumba-Nuñez TI. Beliefs and nutritional practice in pregnant women. Acta Méd Domin 1994; 16(1):5-7.

6. México. Norma Oficial Mexicana NOM-043-SSA22012, Servicios básicos de salud. Promoción y educación para la salud en materia alimentaria. Criterios para brindar orientación. DOF 2013. 
7. Sánchez-Jiménez B, Sámano-Sámano R, PinzónRivera I, Balas-Nakash M, Perichart-Perera O. Factores socioculturales y del entorno que influyen en las prácticas alimentarias de gestantes con y sin obesidad. Revista de Salud Pública y Nutrición RESPYN 2010; 11(2). [Internet]. [consultado 2013 ago 22]. Disponible en: http://www.medigraphic. com/pdfs/revsalpubnut/spn-2010/spn102c.pdf

8. Busdiecker BS, Castillo DC, Salas AI. Cambios en los hábitos de alimentación durante la infancia: una visión antropológica. Rev Chil Pediatr 2000; 71(1):5-11.

9. Langer A. El embarazo no deseado: impacto sobre la salud y la sociedad en América Latina y el Caribe. Temas de Actualidad. Rev Panam Salud Publica 2002; 11(3):192-205.

10. Barquera S, Rivera JA, Espinoza-Montero J, Safdie M, Campirano F, Monterrubio E. Energy and nutrient consumption in Mexican women 12-49 years of age: Analysis of the National Nutrition Survey, 1999. Salud Publica Mex 2003; 45(Supl. 4):S530-S539.

11. Pérez-Gil Romo SE. Antropología, género, alimentación y nutrición en México. Tempo (Revista Casa del Tiempo UAM). [Internet]. [consultado 2013 agosto 22]. Disponible en: http://www.uam.mx/difusion/ revista/dic2000/perez.html

12. Khan R, Blum LS, Sultana M, Bilkis S, Koblinsky M. An examination of women experiencing obstetric complications requiring emergency care: perceptions and sociocultural consequences of caesarean sections in Bangladesh. J Health Popul Nutr 2012; 30(2):159-171.

13. Quintero-Ángel D. Alimentación familiar: una mirada a la cotidianidad en un barrio de clase media en Cali (Colombia). Revista Sociedad y Economía 2008; 14(junio):60-81

14. Gracia-Arnaíz M. ¿Qué hay para comer?: alimentación cotidiana, trabajo doméstico y relaciones de género. Revista Caderno Espaço Femenino 2009; 21(1):209-237.

15. Paul JA, Frings-Dressen MHW. Standing working posture compared in pregnant and nonpregnant conditions. Ergonomics 1994; 37(9):1563-1575.

16. Sally Ede O, Anjos LA, Wahrlich V. Basal metabolism during pregnancy: a systematic review. Cien Saude Colet 2013; 18(2):413-430.

17. Taylor SJ, Bodgan R. Introducción a los métodos cualitativos de investigación. Barcelona: Paidos Básica; 1998.

18. Donoso B, Oyarzún E. High risk pregnancy. Medwave 2012; 12(5):e5429.

19. Guest G, Bunce A, Johnson L. How many interviews are enough: an experiment with data saturation and variability. Field Methods 2006; 18(1):58-82.

20. Bardin L. Análise de Conteúdo. Lisboa: Edições 70; 1977.

21. Luna-Matos ML, Salinas-Piélago J, Luna-Figueroa A. Depresión mayor en embarazadas atendidas en el Instituto Nacional Materno Perinatal de Lima, Perú. Rev Panam Salud Pública 2009; 26(4):310-314.

22. Nazar-Beutelspacher A, Salvatierra-Izaba B. Embarazo no planeado en población indígena y mestiza de asentamientos urbanos marginales de Chiapas. Población y Salud en Mesoamérica 2008; 5(2):1-16.
23. Maroto-Navarro G, Castaño-López E, García-Calvente MM, Hidalgo-Ruzzante N, Mato-Rodríguez I. Paternidad y servicios de salud. Estudio cualitativo de las experiencias y expectativas de los hombres hacia la atención sanitaria del embarazo, parto y posparto de sus parejas. Rev Esp Salud Pública 2009; 83(2):267-278.

24. Rubarth LB, Schoening AM, Cosimano A, Sandhurst H. Women's experience of hospitalized bed rest during high-risk pregnancy. J Obstet Gynecol Neonatal Nurs 2012; 41(3):398-407.

25. Castro-Sánchez AE. La nutrición como ruptura cultural: la experiencia de los adultos con diabetes mellitus tipo 2. Investigación en Salud 2007; IX(1):26-33.

26. Hernández-Sánchez J, Forero-Bulla CM. Conceptions and perceptions on health, quality of life and physical activity in an academia health unit. Rev Cub Enfer 2011; 27(2):159-170.

27. Herrera-Suárez CC, Vásquez-Garibay E, RomeroVelarde E, Romo-Huerta HP, García De Alba García J, Troyo-Sanromán R. Hábitos de alimentación y factores culturales en adolescentes embarazadas. Arch Latinoam Nutr 2008; 58(1):19-26.

28. Mania M, Wojciechowska-Mazurek M, Starska K, Rebeniak M, Postupolski J. Fish and seafood as a source of human exposure to methylmercury. Rocz Panstw Zakl Hig 2012; 63(3):257-264.

29. Chávez Alvarez RE, Arcaya Moncada MJ, Garcia Arias G, Surca Rojas TC, Infante Contreras MV. Rescuing the self-care of health during pregnancy, the delivery and to the newborn: social representations from women of a native community in Peru. Contexto Enferm 2007; 16(4):680-687.

30. Solís-Flores L, Hernández-Rosas JA, Quintero-Estudillo P, Pérez-Garmendia JL, Cortés-Poza D, LópezAlvarenga JC. La consejería personalizada dirigida a mujeres embarazadas disminuye el riesgo de complicaciones secundarias a hipertensión. Informe preliminar. Rev Med Hosp Gen Mex 2009; 72(4):200-206.

31. Thangaratinam S, Rogoziñska E, Jolly K, Glinkowski S, Duda W, Borowiack E, Roseboom T, Tomlinson J, Walczak J, Kunz R, Mol BW, Coomarasamy A, Khan KS. Interventions to reduce or prevent obesity in pregnant women: a systematic review. Health Technol Assess 2012; 16(31):iii-iv, 1-191.

32. Pérez-Gil SE, Vega-García A, Romero-Juárez G. Prácticas alimentarias de mujeres rurales ¿una nueva percepción del cuerpo? Salud Publica Mex 2007; 49(1):52-62.

33. Fonseca AB, De Souza TS, Frozi DS, Pereira RA. Dietary modernity and food consumption: socioanthropological contributions to research in nutrition. Cien Saude Colet 2011; 16(9):3853-3862.

34. Kitchin B. Nutrition counselling for patients with osteoporosis: a personal approach. J Clin Densitom 2013; 16(4):426-431.

35. Furness PJ, McSeveny K, Arden MA, Garland C, Dearden AM, Soltani H. Maternal obesity support services: a qualitative study of the perspectives of women and midwives. BMC Pregnancy Childbirth 2011; 11(69):2-11.

Artigo apresentado em 27/08/2013

Aprovado em 12/12/2013

Versão final apresentada em 16/12/2013 